\title{
On The Composite Ideal
}

\author{
Denik Agustito, Puguh Wahyu Prasetyo, Esti Harini, Istiqomah, Tri Astuti Arigiyati, Irham \\ Taufiq \\ \{rafaelagustito@gmail.com ${ }^{1}$,puguh@pmat.uad.ac.id ${ }^{2}$, estiharini@ustjogja.ac.id ${ }^{3}$ \} \\ Universitas Sarajanawiyata Tamansiswa, Yogyakarta, Indonesia ${ }^{1,3,4,5,6}$, Universitas Ahmad Dahlan \\ Yogyakarta, Indonesia ${ }^{2}$
}

\begin{abstract}
This paperwork in the commutative ring and general results of my research are: ideal $\mathrm{I}$ is called composite if and only if $\mathrm{R} / \mathrm{I}$ is a commutative ring with zero-divisor. And then we have I and $\mathrm{J}$ are the composite ideals of $\mathrm{R}$ such that $\mathrm{I}$ and $\mathrm{J}$ is coprime then $I \cap J$ is a composite ideal of R. If we have $I \subseteq J$ are two ideas of $\mathrm{R}$ and $\mathrm{J}$ is a composite ideal of R then J/I is a composite ideal of R/I.
\end{abstract}

Keywords: Composite, Coprimely, Ideal

\section{Introduction}

Let $\mathrm{R}$ be a commutative ring and a is a non-zero element of $\mathrm{R}$ is called not zero-divisor if there exist non-zero element $\mathrm{b}$ of $\mathrm{R}$ such that $a b \neq 0$. The commutative ring $\mathrm{R}$ is called ring without zero-divisor if for any $a, b \in R$ such that $a, b \neq 0$ then $a b \neq 0$. This is equivalent to saying that the commutative ring $\mathrm{R}$ is called without zero-divisor if-then $a=0$ or $b=0$. From this idea, we have a property from trivial ideal $\{0\}$ is if $a b \in\{0\}$ then $a \in\{0\}$ or $b \in\{0\}$. In a study of Commutative Algebra, the property of trivial ideal ${ }^{\{0\}}$ is generalized into non-trivial ideal as follow: if $a b \in I$ then $a \in I$ or $b \in I$, and then the ideal I is called a prime ideal [1].

Some properties from prime ideal in the study of Commutative Algebra is as follows: Let $R$ be a commutative ring with the unit then $P$ is a prime ideal of $R$ if and only if $R / P$ is a domain integral (Hungerford : 1974). And then we introduce a prime element $\mathrm{p}$ of $\mathrm{R}$ is a nonzero and non-unit element and $\left.p\right|_{a b}$ then $\left.p\right|_{a}$ or $p \mid b$ [2]. If $\mathrm{p}$ is a prime element in commutative ring $\mathrm{R}$ then the principal ideal $(p)=\{p r \mid r \in R\}$ is a prime ideal of $\mathrm{R}$. And then if we have $P_{1}$ and $P_{2}$ is called prime ideals, then $P_{1} \cap P_{2}$ is so.

In a commutative ring $\mathrm{R}$, the non-zero element is denoted by a is called zero-divisor if there exist non-zero element $\mathrm{b}$ such that $a b=0$ [3]. The commutative ring $\mathrm{R}$ is called ring with zero-divisor if there exist two elements $a, b \in R$ where $a, b \neq 0$ but $a b=0$. From this idea we have a property from trivial ideal ${ }^{\{0\}}$ is ${ }^{a, b} \notin\{0\}$ but $a b \in\{0\}$. From the property of this trivial ideal, we can generalize into non-trivial ideal is as follow: If there exist $a, b \in R$ with $a, b \notin I$ but $a b \in I$ then I is called the composite ideal. The purpose of this paper is to find out the characters that must be possessed by a composite ideal in a commutative ring. Referring to the composite ideal 
Idea in a commutative ring, we get an idea of the ideal properties of a composite that is analogous to a composite number. Some things related to the development of composite numbers can be found in the following paper [4] and [5].

\section{Main Results}

In a study of Commutative Algebra, the ideal $\mathrm{P}$ is called prime ideal of a commutative ring if-then $a \in P$ or $b \in P$. Then we introduce a new ideal with property anti-prime is as follow:

\subsection{Definition}

Let $\mathrm{R}$ be a commutative ring dan I is a proper ideal of R. Ideal I si called composite ideal of $\mathrm{R}$ if there exist $a, b \in R, a, b \notin I$ but $a b \in I$.

\subsection{Example}

In the commutative ring $\mathbb{Z}^{\mathbb{Z}}$, the ideal $6 \mathbb{Z}$ is a composite ideal, because we have $4,9 \notin 6 \mathbb{Z}$ but $36=4.9 \in 6 \mathbb{E}$

\subsection{Example}

In the commutative ring $\mathbb{Z}_{20}$, the ideal $l=\{\overline{0}, \overline{10}\}$ is a composite ideal, because we have $\overline{2}, \overline{5} \notin J=\{\overline{0}, \overline{10}\}$ but $\overline{10}=\overline{2}, \overline{5} \in J=\{\overline{0}, \overline{10}\}$.

\subsection{Theorem}

Let ${ }^{n}$ be a non-zero and non-unit element of the commutative ring of an integer number $\mathbb{Z}$. The number $n$ is called composite number if and only if $n \mathbb{Z}$ is a composite ideal of $\mathbb{Z}$.

Proof.

Since $\mathrm{n}$ be a composite number, then $\mathrm{n}$ is not a prime number. So we have there existed $a,\left.b \notin \mathbb{Z}_{\text {where }} n\right|_{a b}$ but $n \nmid a$ and ${ }^{n \nmid b}$. Since there exist ${ }^{a, b} \notin \mathbb{Z}$ where $\left.n\right|_{a b}$ but ${ }^{n \nmid a}$ and $n \nmid b$, then we have there existed ${ }^{a, b \notin \mathbb{Z}}$ where $a b \in n \mathbb{Z}$ but $a \notin n \mathbb{Z}$ and $b \notin n \mathbb{Z}$. So $n \mathbb{Z}$ is a composite ideal of $\mathbb{Z}$.

Conversely, we have $n \mathbb{Z}$ is a composite ideal of $\mathbb{Z}$. It si clear that there exist $a, b \notin \mathbb{Z}$ where $a b \in n \mathbb{Z}$ but $a \notin n \mathbb{Z}$ and $b \notin n \mathbb{Z}$. So we have there existed $a, b \notin \mathbb{Z}$ where $\left.n\right|_{a b}$ but $n \nmid a$ and $n \nmid b$. So $\mathrm{n}$ is not a prime number, and then $\mathrm{n}$ is a composite number.

From the Theorem 1.4, we can generalized a notion about a composite element of the commutative ring as follow: 


\subsection{Definition}

Let $\mathrm{R}$ be a more commutative ring and $q \in R$. Element $\mathrm{q}$ is called a composite element of $\mathrm{R}$ if $\mathrm{q}$ is non-zero and non-unit and there exist $a, b \in R$ where $q \nmid a$ and $q \nmid b$ but $q \mid a b$.

\subsection{Theorem}

Let $\mathrm{R}$ be a commutative ring and $q \in R$. Element $\mathrm{q}$ is called a composite element of $\mathrm{R}$ if and only if the principal ideal $(q)=\{q r \mid r \in R\}$ is a composite ideal of R.

Proof.

Since $\mathrm{q}$ be a composite element, then $\mathrm{q}$ is not a prime number. So we have there existed $a, b \notin R$ where $\mathrm{q}^{l a b}$ but $\mathrm{q}^{\nmid a}$ and $\mathrm{q}^{\nmid b}$. Since there exist $a, b \notin R$ where $q$ lab but $q \nmid a$ and $q \nmid b$, then we have there existed $a, b \notin R$ where $a b \in(q)$ but $a \notin(q)$ and $b \notin(q)$. So $(q)$ is a composite ideal of $R$.

Conversely, we have $(q)$ is a composite ideal of $R$. It is clear that there exist $a, b \notin R$ where $a b \in(q)$ but $a \notin(q)$ and $b \notin(q)$. So we have there existed $a, b \notin R$ where $n \mid a b$ but $n \nmid a$ and ${ }^{n \nmid b}$. So $\mathrm{n}$ is not a prime element, and then $\mathrm{n}$ is a composite element.

The next fact is about the relationship between composite ideal I of a commutative ring with their factor ring.

\subsection{Theorem}

Let $\mathrm{R}$ be a commutative ring and $\mathrm{I}$ is an ideal of $\mathrm{R}$. Factor ring $\mathrm{R} / \mathrm{I}$ is a ring with zerodivisor if and only if $\mathrm{I}$ is a composite ideal.

Proof.

Since factor ring $\mathrm{R} / \mathrm{I}$ is a ring with zero-divisor, then there exist $a+I, b+I \in R / I$ where $a+I \neq I$ and $b+I \neq I$ but $a b+I=I$. So we have there existed $a, b \in R$ where ${ }^{a, b} I$ but $a b \in I$. So ideal $I$ is a composite ideal.

Conversely, since ideal I is a composite ideal, then there exist $a, b \in R$ where $a, b \notin I$ but $a b \in I$. So we have there existed $a+I_{x} b+I \in R / I$ where $a+I \neq I$ and $b+I \neq I$ but $a b+I=I$. It is clear that factor ring $\mathrm{R} / \mathrm{I}$ is a ring with zero-divisor.

\subsection{Corrolary}

The natural number $\mathrm{n}$ is a composite number if and only if $\mathbf{z}_{\mathrm{n}}$ is not an integral domain. Proof.

Since $\mathrm{n}$ is a composite number, based on Theorem 1.4, it is clear that $n \mathbb{E}$ is the composite ideal of ${ }^{\mathbb{Z}}$. Based on Theorem 1.7, so we have $\mathbb{Z}_{n} \cong \mathbb{Z} / n \mathbb{E}$ is not an integral domain.

Conversely, since $\mathbb{Z}_{n}$ is not an integral domain, it is clear $\mathbb{Z}_{n} \cong \mathbb{Z} / n \mathbb{E}$ is not an integral domain. Since $\mathbb{Z} / n \mathbb{E}$ is not an integral domain, based on Theorem 1.7, we have $n \mathbb{E}$ is the composite ideal of ${ }^{\mathbb{Z}}$. Based on Theorem 1.4, we have $\mathrm{n}$ is a composite number. 
Let $\mathrm{R}$ be a commutative ring and $\mathrm{I}, \mathrm{J}$ are ideals of $\mathrm{R}$ and $I \subset J$. The ideal $\mathrm{I}$ is called composite on $\mathrm{J}$ if there exist $a, b \in J$ where $a, b \notin I$ but $a b \in I$. If $l=R$ then I become a composite on $\mathrm{R}$; in another hand I is a composite ideal of $\mathrm{R}$.

\subsection{Lemma}

Let $\mathrm{R}$ be a commutative ring and $\mathrm{I}$, I are ideals of $\mathrm{R}$ such that $I \subset J$. If $\mathrm{I}$ is composite on $\mathrm{J}$ then I is composite on R.

Proof.

Since I is composite ideal on $\mathrm{J}$, it is clear that there exist $a, b \in J$ where ${ }^{a, b} \mathbb{b}$ but $a b \in I$. Since $\mathrm{J}$ is an ideal of $\mathrm{R}$, then we have I is the composite ideal of R. So I is composite on R.

\subsection{Definition}

Let $\mathrm{R}$ be a commutative ring and $\mathrm{I}$, $\mathrm{J}$ are ideals of $\mathrm{R}$. I and $\mathrm{J}$ is said coprime if $I+J=R$.

\subsection{Lemma}

Let $\mathrm{R}$ be a commutative ring. If $\mathrm{I}$ and $\mathrm{J}$ are ideals of $\mathrm{R}$ then there exist ring isomorphism $l /(I \cap j) \cong(I+J) /]$.

Some results of the relationship between coprime between ideals and ring isomorphism theorem (Lemma 1.11) is used to show that intersection between composite ideals is a composite ideal.

\subsection{Theorema}

Let $\mathrm{R}$ be a commutative ring and $\mathrm{I}, \mathrm{J}$ ideal of $\mathrm{R}$ is coprime. If $\mathrm{J}$ is composite on $\mathrm{R}$ then $I \cap J$ is the composite ideal of R.

Proof.

Since ideal, I and $\mathrm{J}$ of $\mathrm{R}$ is coprime, then $I+J=R$. By Lemma 1.11, we have $l /(I \cap J) \cong(I+J) / J=R / J$. Since $\mathrm{J}$ is composite on $\mathrm{R}$, then $R / J$ is not an integral domain, and consequently $l /(I \cap J)$ is not an integral domain. By Theorem 1.7, we have $I \cap J$ is composite on I. By Lemma 1.9, we have $I \cap J$ is the composite ideal of R.

\subsection{Corrolary}

Let $\mathrm{R}$ be a commutative ring and $\mathrm{I}, \mathrm{J}$ are ideals of $\mathrm{R}$ is coprime. If $\mathrm{I}$ and $\mathrm{J}$ are composite ideals then $I \cap J$ is the composite ideal of $\mathrm{R}$.

Proof.

By Theorem 1.12, since $\mathrm{I}$ and $\mathrm{J}$ are ideals of $\mathrm{R}$ is coprime and $\mathrm{I}$ and $\mathrm{J}$ are composite ideals, then it is clear that $I \cap J$ is the composite ideal of R. 


\subsection{Lemma}

Let $\mathrm{R}$ be a commutative ring and $\mathrm{I}, \mathrm{J}, \mathrm{K}$ are ideals of $\mathrm{R}$. If $\mathrm{I}$ and $\mathrm{J}$ is coprime, $\mathrm{J}$ and $\mathrm{K}$ is coprime, and $\mathrm{I}$ and $\mathrm{K}$ is coprime, then $\mathrm{I}$ and $l \cap K$ is coprime.

Proof.

Since $\mathrm{J}$ and $\mathrm{K}$ is coprime, then $l K=J \cap K$ [6]. Since $\mathrm{I}$ and $\mathrm{J}$ is coprime and $\mathrm{I}$ and $\mathrm{K}$ is coprime, then I and $l K=J \cap K$ is coprime [6]. So we have I and $l \cap K$ is coprime.

\subsection{Theorem}

Let $\mathrm{R}$ be a commutative ring and $\mathrm{I}, \mathrm{J}, \mathrm{K}$ are ideals of $\mathrm{R}$. If $\mathrm{I}$ and $\mathrm{J}$ is coprime, $\mathrm{J}$ and $\mathrm{K}$ is coprime, and $\mathrm{I}$ and $\mathrm{K}$ is coprime, then $I \cap J \cap K$ is a composite ideal of $\mathrm{R}$.

Proof.

Since $\mathrm{I}, \mathrm{J}$ and $\mathrm{K}$ are composite ideals of $\mathrm{R}$, $\mathrm{I}$ and $\mathrm{J}$ is coprime, $\mathrm{J}$ and $\mathrm{K}$ is coprime, and $\mathrm{I}$ and $\mathrm{K}$ is coprime, then based on Corrolary 1.13 we have $I \cap J, I \cap K$ and $l \cap K$ are composite ideals of R. Based on Lemma 1.14, we have I and $l \cap K$ is coprime. Since I is a composite ideal of R, based on Corollary 1.13, we have $I \cap J \cap K$ is a composite ideal of R.

\subsection{Lemma}

Let $\mathrm{R}$ be a commutative ring and $\mathrm{I}$ and $\mathrm{J}$ are ideals of $\mathrm{R}$. If $I \subseteq J$ then $(R / I) /(I / I) \cong R / J$.

\subsection{Theorem}

Let $\mathrm{R}$ be a commutative ring and $\mathrm{I}$ and $\mathrm{J}$ are ideals of $\mathrm{R}$. If $I \subseteq J$ and $\mathrm{J}$ is the composite ideal of $\mathrm{R}$ then $\mathrm{J} / \mathrm{I}$ is a composite ideal of factor ring $\mathrm{R} / \mathrm{I}$.

Proof.

Since $I \subseteq J$ and $\mathrm{J}$ is the composite ideal of $\mathrm{R}$, based on Lemma 1.16 and Theorem 1.7 we have $(R / I) /(I / I) \cong R / J$ has a zero-divisor. It is clear that $(R / I) /(I / I)$ has a zero-divisor and based on Theorem 1.7, we have $\mathrm{J} / \mathrm{I}$ is a composite ideal of factor ring R/I.

\section{Conclusion}

Main results in this paper as follow: on the commutative ring $\mathrm{R}$, ideal $\mathrm{I}$ of $\mathrm{R}$ is called composite ideal if and only if the factor ring R/I is ring with zero-divisor. And then if $\mathrm{I}$ and $\mathrm{J}$ are ideals of $\mathrm{R}$ is coprime then $I \cap J$ is the composite ideal of $\mathrm{R}$. If $I \subseteq J$ and $\mathrm{J}$ is the composite ideal of $\mathrm{R}$ then $\mathrm{J} / \mathrm{I}$ is the composite ideal of factor ring $\mathrm{R} / \mathrm{I}$.

\section{References}

[1] W. F and K. H, "Foundations of Commutative Rings and Their Modules," in Springer Nature Singapore, 2016.

[2] H. T. W, Algebra. Springer-Verlag New York. Inc, 1974. 
[3] A. M. F, Introduction to Commutative Algebra. Addison-Wesley Publishing Company. Inc, 1969.

[4] D. D, "Classification and Properties of Composite Numbers with respect to its wings," Int. J. Sci. Eng. Res., vol. 7, no. 2, 2016.

[5] R. S, Highly Composite Numbers. Kluwer academic, 1997.

[6] S. B, Basic Commutative Algebra. World Scientific Publishing Co. Pte. Ltd, 2011. 